\title{
Operational Effectiveness Evaluation of Warship Integrated Communication System
}

\author{
Jiang Zhang \\ Academy of Information \& Communication National University of Defense Technology, Liberation Park Road Wuhan, Hubei \\ Province, China
}

\begin{abstract}
This paper issued how to evaluate the operational effectiveness of warship integrated communication System. At first , it constructed the indicator system with evaluation factors mainly related to operational ability. And then based on the grey relational projection model, it calculated the grey relational projection values of three different application schemes step by step with the method of hierarchical progressive approach. Finally, by comparing three values, the optimal scheme was chosen to be the one whose grey relational projection value was the closest to the ideal value. Practice shows that the calculation method is simple and intuitive, and that it is very suitable for the multi-index evaluation of operational systems.
\end{abstract}

Keywords-warship integrated communication system; grey relational projection model; operational effectiveness; evalvation

\section{INTRODUCTION}

Warship integrated communication system is still in a stage of research and development, in order to be able to demonstrate to the research and development, operational and combat plan simulation training and provide a reliable basis for decisionmaking and reasonable suggestions, it is necessary to evaluating the operational effectiveness of warship integrated communication system, the key of which is to establish a scientific, reasonable, simple and intuitive evaluation and calculation method.

According to the evaluation index system has been established, the method of hierarchical progressive based on gray relational projection theory is proposed for the computation of the evaluation the operational effectiveness of warship integrated communication system in paper.

\section{Evaluation MODEL}

The grey relational projection theory for evaluation is based on a number of quantitative or qualitative index and the preference information of the decision makers, which achieves the ranking for the operational effectiveness of the schemes to be evaluated. The model of grey relational projection theory is as follow:

$$
\left\{\begin{array}{l}
\max _{A_{i} \in A} D_{i} \\
A=\left\{A_{1}, A_{2}, \cdots, A_{n}\right\}
\end{array}\right.
$$

In above formula: $A$ is the set of system construction scheme to be evaluated, $D_{i}$ is the grey relational projection value for evaluation index of system construction scheme $A_{i}$.
According to the calculation results of $D_{i}$, we can judge the effectiveness of each system construction scheme. The higher the value of $D_{i}$, the better the performance of the scheme.

Therefore, the core idea of grey relation projection evaluation is as follow:

- Construct effectiveness vectors of the schemes to be evaluated and ideal scheme by decision attribute matrix;

- $\quad$ Construct a weighted grey relational decision matrix by introducing the correlation function;

- $\quad$ Achieve the relevance of the schemes to be evaluated and ideal scheme according to the weight vector;

- Calculate the grey relational projection values of three different application schemes step by step with the method of hierarchical progressive approach;

- Choose the optimal scheme whose grey relational projection value was the closest to the ideal value.

\section{Calculating Procedure of Evaluation}

After getting the intuitive understanding of the grey relational projection model and the core idea, the calculation process of the grey relational projection model is introduced in detail as follow, according to the evaluation of the secondary index transmission speed for warship integrated communication system.

Based on the analysis on the characteristics of warship integrated communication system, we can evaluate the operational effectiveness of warship integrated communication system from six aspects, such as the reliability and maintainability of system, fast delivery capability, data-link ability, joint distribution capacity, centralized management and control ability, security protection ability.

\section{A. Determination of Decision Attribute Matrix}

First of all, we need to construct the effectiveness vector of scheme to be evaluated by the decision attribute matrix.

It is assumed that there are three kinds of ships integrated communication system needing to be evaluated and calculated, and the data of the 6 evaluation indexes of the transmission speed has been obtained, we can establish a form by taking the evaluation index for the line and the scheme to be evaluated for the column, as shown in Table I, and then get a set of schemes 
to be evaluated $A=\left\{A_{1}, A_{2}, A_{3}\right\}$, and a set of evaluation indexes $V=\left\{V_{1}, V_{2}, V_{3}\right\}$.

TABLE I. TRANSMISSION RATE EVALUATION INDEX

\begin{tabular}{ccccccc}
\hline & \multicolumn{5}{c}{ evaluation index } \\
\cline { 2 - 7 } schemes & B21 & B22 & B23 & B24 & B25 & B26 \\
& $($ Gbps $)$ & $($ Mbps $)$ & $($ Kbps $)$ & $($ Kbps $)$ & $($ Kbps $)$ & $($ bps $)$ \\
\hline $\mathrm{A}_{1}$ & 40 & 300 & 2.4 & 238 & 4 & 12.5 \\
$\mathrm{~A}_{2}$ & 45 & 255 & 1.6 & 165 & 2 & 25.0 \\
$\mathrm{~A}_{3}$ & 35 & 192 & 3.6 & 288 & 1 & 6.25 \\
\hline
\end{tabular}

If the table is summed up, taking the evaluation index for the line and the scheme to be evaluated for the column, we can formula the attribute value table, as shown in Table II.

TABLE II. ATTRIBUTE VALUE TABLE

\begin{tabular}{ccccc}
\hline Scheme & $\mathrm{V}_{1}$ & $\mathrm{~V}_{2}$ & $\cdots$ & $\mathrm{V}_{\mathrm{m}}$ \\
\hline $\mathrm{A}_{1}$ & $\mathrm{x}_{11}$ & $\mathrm{x}_{12}$ & $\cdots$ & $\mathrm{x}_{1 \mathrm{~m}}$ \\
$\mathrm{~A}_{2}$ & $\mathrm{x}_{21}$ & $\mathrm{x}_{22}$ & $\cdots$ & $\mathrm{x}_{2 \mathrm{~m}}$ \\
$\vdots$ & $\vdots$ & $\vdots$ & $\vdots$ & $\vdots$ \\
$\mathrm{A}_{\mathrm{n}}$ & $\mathrm{x}_{\mathrm{n} 1}$ & $\mathrm{x}_{\mathrm{n} 2}$ & $\cdots$ & $\mathrm{x}_{\mathrm{nm}}$ \\
\hline
\end{tabular}

In table 2, the set of schemes are $A=\left\{A_{1}, A_{2}, \cdots, A_{n}\right\}$, the set of evaluation index are $V=\left\{V_{1}, V_{2}, \cdots, V_{m}\right\}$, so that, the value of evaluation index $V_{j}$. in scheme $A_{i}$ is $x_{i j}$, which is the basis for describing the effectiveness vector of schemes to be evaluated.

Assuming that the value of evaluation index $V_{j}$ in ideal scheme is $x_{o j}$, the maximum and minimum values of $x_{o j}$ are selected for different types of indexes, as follow:

$$
x_{0 j}= \begin{cases}\max \left(x_{1 j}, x_{2 j}, \cdots, x_{n j}\right), & V_{j}: \text { efficiency index } \\ \min \left(x_{1 j}, x_{2 j}, \cdots, x_{n j}\right), & V_{j}: \text { cost index }\end{cases}
$$

In above formula, $x_{o j}$ is ready for describing the effectiveness vector of ideal scheme, therefore, we can get the decision attribute matrix $X$ of the indexes set $V$ under the set of schemes $A$, as $X=\left(X_{i j}\right)_{(n+1) \times m}(i=0,1, \cdots, n ; j=1,2, \cdots, m)$.

$$
X=\left[\begin{array}{llllll}
45 & 300 & 3.6 & 288 & 4 & 25.0 \\
40 & 300 & 2.4 & 238 & 4 & 12.5 \\
45 & 255 & 1.6 & 165 & 2 & 25.0 \\
35 & 192 & 3.6 & 288 & 1 & 6.25
\end{array}\right]
$$

\section{B. The Normalization of Decision Attribute Matrix}

After getting the effectiveness vector of schemes to be evaluated, the normalization of decision attribute matrix is the next step in the follow. Because of the difference in type, dimension and quantity for the evaluation indexes of the system, the indexes can't be directly compared. Therefore, in order to ensure the reliability of the evaluation results, it is necessary to deal with the attribute value of the evaluation index in the process of initial value. All data in a sequence are divided by someone of the sequence. So we can get a new sequence, which is called initial value processing method.

Assume that the attribute value after initialization is $y_{i j}$, we can get the efficiency index $y_{i j}=x_{i j} / x_{0 j}$, and the cost index is just the opposite, as follow:

$$
y_{i j}= \begin{cases}x_{i j} / x_{0 j}, & V_{j}: \text { efficiency index } \\ x_{0 j} / x_{i j}, & V_{j}: \text { cost index }\end{cases}
$$

From above formula, it is clear that there is a value $y_{0 j}=1$ for each indicator. After such a process, the ideal scheme of the index value sequence has become the all-one sequence, which can greatly simplify the calculation. According to $y_{i j}$, we can build a standardized decision attribute matrix of index set $V$ for the set of schemes $A$, as $Y=\left(y_{i j}\right)_{(n+1) \times m}$.

$$
Y=\left[\begin{array}{llllll}
1 & 1 & 1 & 1 & 1 & 1 \\
0.8889 & 1 & 0.6667 & 0.8264 & 1 & 0.5 \\
1 & 0.85 & 0.4444 & 0.5729 & 0.5 & 1 \\
0.7778 & 0.64 & 1 & 1 & 0.25 & 0.25
\end{array}\right]
$$

\section{Construction of Grey Relational Judgment Matrix}

After getting the standardized decision attribute matrix, the most important step is to build a grey relational matrix.

According to the definition of relational projection model in grey system theory, we use $y_{0 j}$ as the parent factor and $y_{i j}$ as the sub factor, and the expression of relativity for $f_{i j}$ can be obtained. Therefore, we can construct a grey relational judgment matrix $\mathrm{F}$ basing on the number $(n+1) \times m$ of $f_{i j}$.

$$
F=\left[\begin{array}{llllll}
1 & 1 & 1 & 1 & 1 & 1 \\
0.7714 & 1 & 0.5294 & 0.6835 & 1 & 0.4286 \\
1 & 0.7143 & 0.4029 & 0.4675 & 0.4286 & 1 \\
0.6279 & 0.5102 & 1 & 1 & 0.3333 & 0.3333
\end{array}\right]
$$

So the relationship between the scheme $A_{1}$ to be evaluated and ideal scheme $A_{0}$ is established. 


\section{Establishment of Weighted grey Relational Decision Matrix}

The establishment of weighted grey relational decision matrix is divided into two steps.

In first step, weight judgment matrix A is established. Assuming that each evaluation index is characterized by $\mathrm{n}$ factors $V_{i}$, the relative importance of $V_{i}$ is compared with each other, which achieve the relative importance value $a_{i j}$ of $V_{i}$. Therefore, how to regulate $a_{i j}$ ? Here, the evaluation criteria given by the method of hierarchical progressive approach is needed. According to the evaluation criteria, after comparing all factors with each other, a weighted judgment matrix is established with $a_{i j}$. Usually, we ask some experts to score their geometric mean to determine the final weighted matrix.

In next step, the characteristic value and the characteristic vector of the weight judgment matrix are calculated to get the maximum characteristic value $\lambda_{\max }$ which meets $A W=\lambda_{\text {max }} W$, and the normalized characteristic vector $W=\left\{w_{1}, w_{2}, \cdots, w_{n}\right\}$. If the weighted judgment matrix could be tested by the consistency then the component $w_{i}$ of $W$ can be used as the weighted value of the corresponding evaluation index $V_{i}$.

Assume that the weighted vector between the evaluation indexes is $W=\left(w_{1}, w_{2}, L w_{m}\right)^{T}$, the weighted grey relational decision matrix $F^{\prime}$ can be established, and whose row vector is the sequence for describing effectiveness vector of the ideal scheme and the schemes to be evaluated. At this point, we get a complete representation of relational expression between $A_{1}$ and $A_{0}$, as follow.

$$
F^{\prime}=F \cdot W=\left[\begin{array}{ccccc}
1 & 1 & 1 & \cdots & 1 \\
f_{11} & f_{12} & f_{13} & \cdots & f_{1 m} \\
f_{21} & f_{22} & f_{23} & \cdots & f_{2 m} \\
\vdots & \vdots & \vdots & & \vdots \\
f_{n 1} & f_{n 2} & f_{n 3} & \cdots & f_{n m}
\end{array}\right]\left[\begin{array}{c}
w_{1} \\
w_{2} \\
\vdots \\
w_{m}
\end{array}\right]=\left[\begin{array}{c}
A_{0} \\
A_{1} \\
A_{2} \\
\vdots \\
A_{m}
\end{array}\right]
$$

\section{E. Calculation of the Grey Relational Projection Value}

According to the formula for solving grey relational projection value $D_{i}$, and the effectiveness vector expression for the scheme based on the weighted grey relational decision matrix, the calculation formula of $D_{i}$ based on index relational degree and weight can be obtained. If the index weighted vector is normalized, and the normalized $\overline{W_{j}}$ is the grey relational projection vector, $D_{i}$ can be simply expressed as the product of two vectors, as follow.

$$
D_{i}=\sum_{j=1}^{m} F_{i j} \overline{W_{j}}, \quad i=1,2, \cdots, m
$$

Taking the data into the calculation of formula (8), then we get as follow:

$$
\left[\begin{array}{l}
D_{1} \\
D_{2} \\
D_{3}
\end{array}\right]=\left[\begin{array}{l}
0.6585 \\
0.9406 \\
0.5027
\end{array}\right]
$$

If only the transmission speed of the system is evaluated, then our evaluation calculation is completed. According to the score, it is clear that the transmission speed of the second scheme is the most excellent.

\section{F. Calculation by Hierarchical Progressive Approach}

If we want to evaluate the operational effectiveness of the warship integrated communication system, we need to use the grey relational projection method. Its calculation steps are:

1. The grey relational projection value of all secondary indexes of the scheme to be evaluated is calculated, according to the evaluation procedure of the grey relational projection method.

2. The result above is used as the evaluation data of the first level index to calculate the grey relational projection value of the first level indexes.

For example, assuming that we have completed the calculation of the grey relational projection value of the secondary indexes transmission rate and the transmission quality of the three schemes to be evaluated, by taking these results as the index values, we can evaluate the first level evaluation index the fast transmission capability to get the grey relational projection value, as shown in Figure I.

$\begin{array}{ccc}\hline \multirow{2}{*}{\text { Schemes }} & \multicolumn{2}{c}{\text { Evaluation Indexes of Fast Transmission }} \\$\cline { 2 - 3 } & \text {$Transmission rate } & \text { Transmission quality } \\$\cline { 2 - 3 } $\left.\mathbf{A}_{1} & \mathbf{D}_{11} & \mathbf{D}_{12} \\ \mathbf{A}_{2} & \mathbf{D}_{21} & \mathbf{D}_{22} \\ \mathbf{A}_{3} & \mathbf{D}_{31} & \mathbf{D}_{32}\end{array}\right]\left[\begin{array}{c}D_{1 B} \\ D_{2 B} \\ D_{3 B}\end{array}\right]$

FIGURE I. THE FIRST LEVEL INDEXES ARE EVALUATED BY HIERARCHICAL PROGRESSIVE METHOD BASED ON THE SECONDARY INDEXES GREY PROJECTION VALUE

3. Taking the grey relational projection value of the first level index as the index data of the system effectiveness, and the system level grey relational projection value of is calculated, according to which, we can compare and sequence the operational effectiveness $\mathrm{S}$ for each warship integrated communication system. Finally, according to the results of sequence, analysis and suggestion is given, and at this point we complete the whole evaluation calculation, as shown in Figure II. 


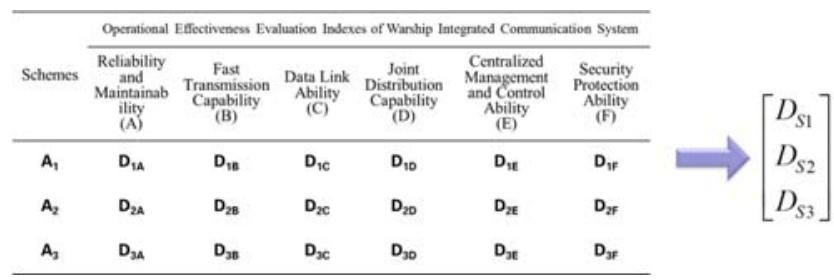

FIGURE II. EVALUATION OF THE SYSTEM LEVEL INDEXES BY HIERARCHICALPROGRESSIVEMETHOD

\section{CONCLUSION}

Operational effectiveness evaluation of warship integrated communication system is still in a stage of exploration, to get a real and effective evaluation results, the complete evaluation index system, practical index weight, real effective index data and the simple evaluation model are indispensable. The grey relational projection evaluation model based on grey theory not only considers the impact of a variety of indexes, but also realizes the progressive calculation according to the hierarchical relationship of the index system, and eventually achieves the integrated communication system effectiveness scoring sequence. The idea of this model is reasonable, the calculation process is clear and the calculation method is simple and convenient. It is a very effective evaluation model, which has important practical value to improve the operational effectiveness evaluation of the warship integrated communication system.

\section{REFERENCES.}

[1] G. Eason, B. Noble, and I. N. Sneddon, "On certain integrals of Lipschitz-Hankel type involving products of Bessel functions,” Phil. Trans. Roy. Soc. London, vol. A247, pp. 529-551, April 1955. (references)

[2] J. Clerk Maxwell, A Treatise on Electricity and Magnetism, 3rd ed., vol. 2. Oxford: Clarendon, 1892, pp.68-73.

[3] I. S. Jacobs and C. P. Bean, "Fine particles, thin films and exchange anisotropy,” in Magnetism, vol. III, G. T. Rado and H. Suhl, Eds. New York: Academic, 1963, pp. 271-350.

[4] K. Elissa, "Title of paper if known," unpublished.

[5] R. Nicole, "Title of paper with only first word capitalized," J. Name Stand. Abbrev., in press.

[6] Y. Yorozu, M. Hirano, K. Oka, and Y. Tagawa, "Electron spectroscopy studies on magneto-optical media and plastic substrate interface,” IEEE Transl. J. Magn. Japan, vol. 2, pp. 740-741, August 1987 [Digests 9th Annual Conf. Magnetics Japan, p. 301, 1982].

[7] M. Young, The Technical Writer's Handbook. Mill Valley, CA: University Science, 1989. 